\title{
Low-symmetry topological materials for large charge-to-spin interconversion: The case of transition metal dichalcogenide monolayers
}

\author{
Marc Vila, ${ }^{1,2}$ Chuang-Han Hsu, ${ }^{3,4}$ Jose H. Garcia $\odot,{ }^{1}$ L. Antonio Benítez $\odot,{ }^{1,2,5}$ Xavier Waintal $\odot,{ }^{6}$ Sergio O. Valenzuela $\odot,{ }^{1,7}$ \\ Vitor M. Pereira $\odot,{ }^{4}$ and Stephan Roche $\odot^{1,7}$ \\ ${ }^{1}$ Catalan Institute of Nanoscience and Nanotechnology (ICN2), CSIC and BIST, Campus UAB, Bellaterra, O8193 Barcelona, Spain \\ ${ }^{2}$ Department of Physics, Universitat Autònoma de Barcelona, Campus UAB, Bellaterra, 08193 Barcelona, Spain \\ ${ }^{3}$ Department of Electrical and Computer Engineering, National University of Singapore, Singapore 117576, Singapore \\ ${ }^{4}$ Centre for Advanced 2D Materials and Graphene Research Centre, National University of Singapore, Singapore 117546, Singapore \\ ${ }^{5}$ Department of Physics, Massachusetts Institute of Technology, Cambridge, Massachusetts 02139-4307, USA \\ ${ }^{6}$ Université Grenoble Alpes, CEA, IRIG-PHELIQS, 38000 Grenoble, France \\ ${ }^{7}$ ICREA-Institució Catalana de Recerca i Estudis Avançats, 08010 Barcelona, Spain
}

(Received 10 June 2020; revised 29 November 2021; accepted 2 December 2021; published 30 December 2021)

\begin{abstract}
The spin polarization induced by the spin Hall effect (SHE) in thin films typically points out of the plane. This is rooted on the specific symmetries of traditionally studied systems, not in a fundamental constraint. Recently, experiments on few-layer $\mathrm{MoTe}_{2}$ and $\mathrm{WTe}_{2}$ showed that the reduced symmetry of these strong spin-orbit coupling materials enables a new form of canted spin Hall effect, characterized by concurrent in-plane and out-of-plane spin polarizations. Here, through quantum transport calculations on realistic device geometries, including disorder, we predict a very large gate-tunable SHE figure of merit $\lambda_{s} \theta_{x y} \approx 1-50 \mathrm{~nm}$ in $\mathrm{MoTe}_{2}$ and $\mathrm{WTe}_{2}$ monolayers that significantly exceeds values of conventional SHE materials. This stems from a concurrent long spin diffusion length $\left(\lambda_{s}\right)$ and charge-to-spin interconversion efficiency as large as $\theta_{x y} \approx 80 \%$, originating from momentum-invariant (persistent) spin textures together with large spin Berry curvature along the Fermi contour, respectively. Generalization to other materials and specific guidelines for unambiguous experimental confirmation are proposed, paving the way toward exploiting such phenomena in spintronic devices. These findings vividly emphasize how crystal symmetry and electronic topology can govern the intrinsic SHE and spin relaxation, and how they may be exploited to broaden the range and efficiency of spintronic materials and functionalities.
\end{abstract}

DOI: 10.1103/PhysRevResearch.3.043230

\section{INTRODUCTION}

Unconventional manifestations of spin-orbit coupling (SOC) are rapidly extending the ability to generate, control, and carry spin polarization for applications of spin transport or spin-driven magnetic torques beyond conventional spintronic materials [1-4]. Topological materials form a natural family to scrutinize in this regard: Their key features often derive from a large SOC combined with band inversions, and their topologically protected surface states may prove instrumental to enable coherent, dissipationless spin currents over long-distances [5,6]. Three-dimensional (3D) Weyl semimetals (WSM) are defined by the presence of band degeneracy points near the Fermi energy $\left(E_{F}\right)$ with local linear dispersion in all directions [7,8]. Layered transition metal dichalcogenides (TMDs) in the $1 \mathrm{~T}^{\prime}\left(P 2_{1} / m\right)$ or $1 \mathrm{~T}_{\mathrm{d}}\left(P m n 2_{1}\right)$ phases accommodate the interesting class of WSM candidates $M X_{2}$

Published by the American Physical Society under the terms of the Creative Commons Attribution 4.0 International license. Further distribution of this work must maintain attribution to the author(s) and the published article's title, journal citation, and DOI.
( $M=\mathrm{Mo}, \mathrm{W} ; X=\mathrm{S}, \mathrm{Se}, \mathrm{Te})$, which have been advanced as platforms for realizing exotic phenomena such as topological superconductivity [9-12], nonlinear Hall effect [13-17], anisotropic spin Hall transport [18], and out-of-plane spinorbit torque $[19,20]$. When thinned toward the monolayer limit, they transition from the type-II WSM bulk phase to the quantum spin Hall regime [21-35] with strain-tunable topological gap [36].

Recently, large charge-to-spin interconversion (CSI) generated by the spin Hall effect (SHE) has been reported in multilayers of $\mathrm{MoTe}_{2}$ and $\mathrm{WTe}_{2}$ [37-40]. The CSI efficiency is quantified in terms of the spin Hall angle (SHA, $\theta_{x y}$ ), which indicates what fraction of a driving charge current $\left(\boldsymbol{J}_{c}\right)$ can be converted into spin current $\left(\boldsymbol{J}_{s}^{\alpha}\right) ; \theta_{x y}$ depends on the magnitude of SOC and is typically no more than a few percent at room temperature in heavy metals [41]. In traditional SHE materials, stronger SOC correlates with shorter spin diffusion length $\left(\lambda_{s}\right)$; consequently, achieving long $\lambda_{s}$ concurrently with large SHA is a long-standing challenge for spintronics. To date, the best tradeoff obtained with heavy metals amounts to $\lambda_{s} \theta_{x y} \approx 0.1-0.2 \mathrm{~nm}[41-46]$.

Interestingly, hints of unconventional SHE have been detected in $1 \mathrm{~T}^{\prime}$ [37] and $1 \mathrm{~T}_{\mathrm{d}}$ phases of $\mathrm{MoTe}_{2}$ multilayers [38], characterized by spin currents carrying spins $(\boldsymbol{S})$ collinear 
with the charge current, which is unique so far. This is possible because, in contrast to bulk crystals, the absence of the glide mirror symmetry in few-layer slabs allows for additional nonzero components of the spin Hall conductivity (SHC) tensor [47], thus breaking away from the traditional constraint imposing a stringent right-hand rule $\boldsymbol{J}_{c} \perp \boldsymbol{J}_{s} \perp \boldsymbol{S}$ ). Furthermore, a remarkably long spin diffusion length $\left(\lambda_{s}\right)$ has been reported in $\mathrm{MoTe}_{2}$ [38], although its actual magnitude is under debate $[37,39,40]$. These experimental developments call for an understanding of the mechanisms governing spin dynamics in such systems, especially the large CSI efficiency $\theta_{x y}$ and what enables large $\lambda_{s}$, given that these are traditionally anticorrelated quantities.

While topological materials could be expected to display large SHE efficiency, primarily driven by a strong SOC and a large intrinsic SHC, here we show that, in addition, the reduced symmetry plays a central role by enabling otherwiseforbidden persistent (i.e., $\boldsymbol{k}$-invariant) spin textures (PST) that sustain unusually large spin diffusion lengths. Specifically, we show that unique symmetry-induced spin textures of electronic states in $\mathrm{MoTe}_{2}$ and $\mathrm{WTe}_{2}$ monolayers yield a giant canted SHE where the spin current polarization lies in the $y z$ plane. CSI efficiencies can be as high as $80 \%$ and values of $\lambda_{s}$ in the range $10-100 \mathrm{~nm}$, up to one order of magnitude larger than in heavy metals with similar spin Hall efficiency [41]. We unveil that these large values arise from the interplay of a PST, confirmed by density functional theory (DFT), and a large spin Berry curvature (SBC), stemming from the band inversion and hybridization near $E_{F}$ that underlies the nontrivial topology of these monolayers. Moreover, all spin transport characteristics are gate tunable, being maximal near the band edge and allowing a CSI figure of merit of up to $\lambda_{s} \theta_{x y} \approx 50 \mathrm{~nm}$. Our findings hinge on DFT, symmetry considerations, and a purpose-built effective tight-binding model deployed in spintronic simulations using state-of-the-art quantum transport methodologies [48]. Importantly, we discuss how similarly large spintronic figures of merit can be anticipated, based on the same key physical ingredients, among several other classes of materials that would be interesting to explore. This paves the way to uncovering systems endowed with both large $\theta_{x y}$ and large $\lambda_{s}$, breaking free from a challenging constraint that has hindered spintronic applications.

\section{THEORETICAL MODEL}

We computed the bandstructures of $1 \mathrm{~T}_{\mathrm{d}}$-derived monolayers of $\mathrm{MoTe}_{2}$ and $\mathrm{WTe}_{2}$ within DFT (see the Supplemental Material [49]). Effective Hamiltonians based on maximally localized Wannier functions were subsequently extracted, allowing straightforward computation of the SHC and spin textures with no intervening approximations. Yet, such a Hamiltonian is still too complex to be efficiently deployed in large-scale transport calculations on system sizes involving millions of unit cells. We therefore built a $\boldsymbol{k} \cdot \boldsymbol{p}$ Hamiltonian to describe the two conduction and two valence bands nearest $E_{F}$ which, at the $\Gamma$ point, transform according to the representations $B_{u}$ (valence) and $A_{g}$ (conduction) of the $C_{2 \mathrm{~h}}$ point group [23] (see the Supplemental Material [49] for further discussion). Extension of the symmetry-allowed $\boldsymbol{k} \cdot \boldsymbol{p}$ terms to the full Brillouin zone yields the following nearest-neighbor tight-binding representation [49]:

$$
\begin{aligned}
H= & \sum_{i, s}\left(\Delta+4 m_{p}+\delta\right) c_{i, s}^{\dagger} c_{i, s}-\sum_{\langle i j\rangle, s}\left(m_{p}+m_{d}\right) c_{i, s}^{\dagger} c_{j, s} \\
& +\sum_{i, s}\left(\Delta-4 m_{d}-\delta\right) d_{i, s}^{\dagger} d_{i, s}-\sum_{\langle i j\rangle, s}\left(m_{p}-m_{d}\right) d_{i, s}^{\dagger} d_{j, s} \\
& -\sum_{\langle i j\rangle, s} \frac{\beta}{2}\left(\hat{\boldsymbol{l}}_{i j} \cdot \hat{\boldsymbol{y}}\right) c_{i, s}^{\dagger} d_{j, s}+\sum_{i, s} \eta c_{i, s}^{\dagger} d_{i, s} \\
& -\sum_{\langle i j\rangle} \sum_{s s^{\prime}} \frac{i}{2}\left(\boldsymbol{\Lambda}_{s s^{\prime}} \times \hat{\boldsymbol{l}}_{i j}\right) \cdot(\hat{\boldsymbol{y}}+\hat{z}) c_{i, s}^{\dagger} d_{j, s^{\prime}}+\text { H.c. }
\end{aligned}
$$

This is an effective four-band Hamiltonian generated by two orbitals (plus spin) per unit cell on a rectangular lattice, one arising from the chalcogen $p_{y}$ states and the other from metal $d_{y z}$ orbitals, respectively associated with the $c_{i, s}$ and $d_{i, s}$ operators at each unit cell $i$ ( $s$ labels the spin projection). The first four terms in Eq. (1) describe spin-degenerate valence and conduction bands with hopping amplitudes set by $m_{p} \pm m_{d}$, $\delta$ parameterizes the degree of band inversion at $\Gamma$, and a constant $\Delta$ is used to match the position of the conduction band and $E_{F}$ with those obtained by DFT. In the fifth term, $\beta$ accounts for the $x-y$ crystalline anisotropy, with $\hat{\boldsymbol{l}}_{i j}$ a unit vector pointing from site $i$ to $j$; the term $\propto \eta$ breaks inversion symmetry and determines, for example, whether we are describing a monolayer descended from a $1 \mathrm{~T}^{\prime}(\eta=0)$ or $1 \mathrm{~T}_{\mathrm{d}}$ bulk crystal. The last term embodies the SOC, where $\boldsymbol{\Lambda} \equiv$ $\left(\Lambda_{x} \sigma_{x},-\Lambda_{y} \sigma_{y}, \Lambda_{z} \sigma_{z}\right), \sigma_{x, y, z}$ are the spin Pauli matrices and $\hat{\boldsymbol{y}}$, $\hat{z}$ are unit Cartesian vectors. The parameters are set by fitting the energy dispersion and spin texture to the ones obtained by DFT [49]. To be specific, we henceforth concentrate on the case of $\mathrm{MoTe}_{2}$, as it is the one where experiments have recently reported in-plane SHE [37,38]. Nonetheless, because the Hamiltonian model (1) captures equally well the case of $\mathrm{WTe}_{2}$ and similar low-symmetry TMDs [49], qualitatively comparable results can be expected in those monolayers as well. Additionally, we found very similar results between the $1 \mathrm{~T}_{\mathrm{d}}$ and $1 \mathrm{~T}^{\prime}$ phases and therefore we focus here on the former while the latter is reported in the Supplemental Material [49]. We favoured the conduction band in the fits and will focus exclusively on cases where $E_{F}$ lies in the conduction band.

\section{NUMERICAL SPIN DYNAMICS CALCULATIONS}

In Fig. 1(a), we plot the band structure of the aforementioned four-band model near $E_{F}$ and one of the time-reversalsymmetric $Q$ points, where the valence and conduction extrema occur. The small splitting arises from the small inversion-symmetry breaking $(\eta \neq 0)$ that occurs in monolayers derived from the $T_{d}$ bulk structure. Since this work focuses on the scenario where $E_{F}$ lies in the conduction band, the figure shows a closeup of the conduction electron pockets; the full DFT band structure and the $\boldsymbol{k} \cdot \boldsymbol{p}$ fit are discussed in the Supplemental Material [49]. Figure 1(b) shows the spin texture at the Fermi energy, $\left\langle s^{\alpha}\right\rangle_{E_{\mathrm{F}}}$, with two crucial observations: the existence of an approximate persistent spin texture through the whole Fermi contour [50-52] and canted spins with $\left\langle s^{y}\right\rangle_{E_{\mathrm{F}}} \gtrsim\left\langle s^{z}\right\rangle_{E_{\mathrm{F}}} \gg\left\langle s^{x}\right\rangle_{E_{\mathrm{F}}}$, consistent with prior studies [15,53]. 

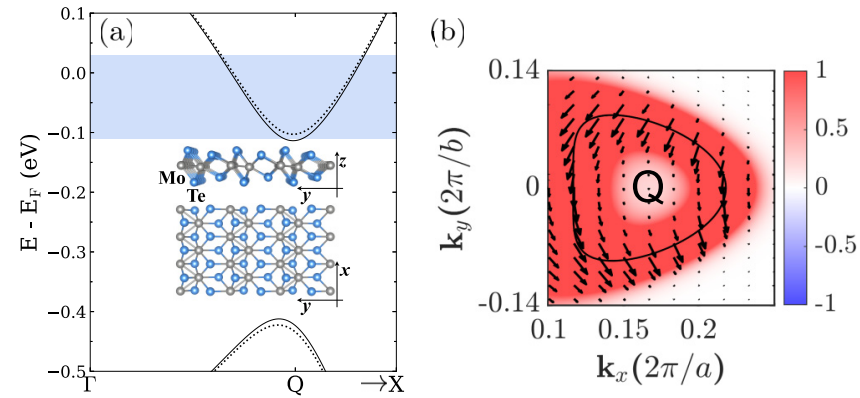

FIG. 1. (a) Closeup of the band structure near $E_{F}$ for the fourband model [Eq. (1)] of $\mathrm{T}_{\mathrm{d}}-\mathrm{MoTe}_{2}$ monolayer near one of the time-reversal-symmetric $Q$ points. The blue-shaded region indicates the energy range covered in the spin transport calculations. Inset: monolayer crystal structure. (b) Spin texture of one of the bands of the electron pocket near Q at $E_{F}$ (Fermi broadened with $T=300 \mathrm{~K}$ ); the solid line marks the Fermi contour, arrows depict the in-plane spin projection, and the color indicates the spin projection along $z$.

We explored the spin transport properties using linear response theory and the Landauer-Büttiker formalism as implemented in KWANT [54]. We simulated the nonlocal spin valve illustrated in the inset of Fig. 2, where contacts 2 and 3 are ferromagnetic (FM) to allow injection and detection of spin-polarized currents [55-57]: FM electrode 2 injects a spinpolarized current $I_{0}^{\alpha}$ with spins polarized along $\alpha \in\{x, y, z\}$; this creates a spin accumulation that diffuses along the channel and is detected as a nonlocal voltage $V_{\mathrm{nl}}$ at electrodes 3 and 4 , located a distance $L$ from the source and far from the path of charge current between electrodes 1 and 2. This effect is quantified by the nonlocal resistance $R_{\mathrm{nl}}^{\alpha} \equiv V_{\mathrm{nl}} / I_{0}^{\alpha}$. The spin diffusion length for $\alpha$-pointing spins, $\lambda_{s}^{\alpha}$, is obtained from the decay of $R_{\mathrm{nl}}^{\alpha}$ with $L$ in the diffusive regime (mean free path shorter than $L$ ). To ensure our results reflect the diffusive

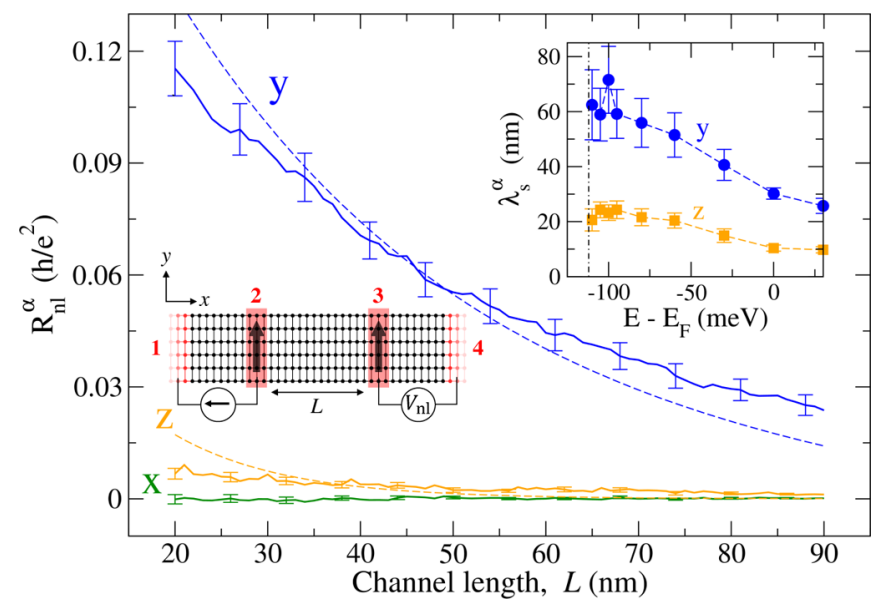

FIG. 2. $R_{\mathrm{nl}}^{\alpha}$ (solid lines) against the channel length, $L$, for spins polarized along $x, y$, and $z$. Error bars result from averaging over 150 disorder configurations $(w=50 \mathrm{~nm})$. Dashed lines are fits to Eq. (10) in Ref. [49]. Left inset: Scheme of the nonlocal spin valve. Black (red) regions denote the device (leads), with leads 2 and 3 being ferromagnetic. Current $I_{0}^{\alpha}$ flows from lead 2 to 1 and $V_{\mathrm{nl}}$ is measured between leads 3 and 4. Right inset: Energy dependence of $\lambda_{s}^{y, z}$. The dot-dashed line marks the conduction band minimum. regime, we add Anderson disorder to the Hamiltonian and extract statistics only within the appropriate scaling region of the device conductance $[49,58]$.

Figure 2 shows $R_{\mathrm{nl}}^{\alpha}(L)$ for the three spin orientations at $E_{F}$. We see clear differences in the magnitude of the nonlocal signals and their relaxation distances for different orientations of the injected spins, ranging from tens of nanometers to the subnanometer scale. By fitting the length dependence of $R_{\mathrm{nl}}^{\alpha}$ to the solution of the spin diffusion equations (dashed lines in Fig. 2) [58], we obtained $\lambda_{s}^{y} \approx 30 \mathrm{~nm}$ and $\lambda_{s}^{z} \approx 10 \mathrm{~nm}$, while $\lambda_{s}^{x}$ has a negligible value. These values are comparable with strong-SOC metals such as $\mathrm{Pt}, \beta-\mathrm{W}$ or $\beta$-Ta $[41,43]$. It is significant that the spin diffusion lengths follow the trend $\lambda_{s}^{y} \gtrsim \lambda_{s}^{z} \gg \lambda_{s}^{x}$, in correspondence to that of the spin texture around the Fermi contour at equilibrium. The upper inset of Fig. 2 shows that this hierarchy holds over the entire range of energies analyzed, from $E=30 \mathrm{meV}$ to the band edge at $\approx-110 \mathrm{meV}$ (we measure energies relative to $E_{F}$ of undoped $\mathrm{MoTe}_{2}$ ). Details of how the (persistent) spin texture impacts $\lambda_{s}^{\alpha}$ and its scaling with energy are discussed in the Supplemental Material [49]. Both $\lambda_{s}^{y}$ and $\lambda_{s}^{z}$ increase about threefold as $E_{F}$ moves toward the band edge (dot-dashed line). Moreover, we found that $\lambda_{s}^{y}$ at $E_{F}=-140 \mathrm{meV}$ (in the band gap) increases up to $\simeq 156 \mathrm{~nm}$, while deeper into the gap $(E=-320 \mathrm{meV})$ we see no decay in the spin signal, consistent with the onset of ballistic regime where spin is transported by topologically protected surface states [49].

The SHE was investigated by computing the spin accumulation and analyzing its polarization, $s^{\alpha}$, along the three Cartesian directions $\alpha$. Charge current along $y$ generates a transverse spin current parallel to $x$ by the SHE, which results in spin accumulation at the open lateral boundaries. The efficiency of CSI is characterized by the SHA, defined as the ratio $\theta_{i j}^{\alpha} \equiv J_{s, i}^{\alpha} / J_{c, j}$, where $\hbar J_{s}^{\alpha} / 2 e\left(\boldsymbol{J}_{c}\right)$ is the spin (charge) current density, $e$ is the electron charge, and $i, j \in\{x, y\}$ denote the respective current directions. To numerically determine the SHA, we calculated the spin accumulation response function per unit of current applied to the lead and fit it to the solution of the spin drift-diffusion equations:

$$
\frac{s^{\alpha}(x)}{J_{c, y}}=-\frac{\theta_{x y}^{\alpha} \lambda_{s}^{\alpha}}{|e| D_{s}} \frac{\sinh \left(\frac{w-2 x}{2 \lambda_{s}^{\alpha}}\right)}{\cosh \left(\frac{w}{2 \lambda_{s}^{\alpha}}\right)},
$$

where $w$ is the device width and $D_{s}$ is the spin diffusion coefficient (see the Supplemental Material [49] for details).

Figure 3 shows the averaged spin accumulation along the channel cross section, $s^{\alpha}(x)$, for each spin orientation. In a typical SHE scenario, the electrical current, spin current, and the spin polarization are all mutually orthogonal (for this geometry, that would generate a finite $s^{z}$ only); however, we observe a nonzero $s^{y}$ as well due to the absence of a glide mirror symmetry in $1 \mathrm{~T}_{\mathrm{d}}-\mathrm{MoTe}_{2}$ monolayer. In fact, $\left|s^{z}\right| \sim\left|s^{y}\right|$, implying that the accumulated spins point obliquely in the $y z$ plane, with significant projection parallel to the electrical current. Interestingly, note that the spin accumulation displays the hierarchy $s^{y} \gtrsim s^{z} \gg s^{x}$, echoing the trend seen above for the spin texture and spin diffusion lengths.

We determine $\theta_{x y}^{\alpha}$ by fitting the numerically calculated spin accumulation to Eq. (2), using the values of $D_{s}$ extracted from the two-terminal conductance of this device and $\lambda_{s}^{\alpha}$ from 


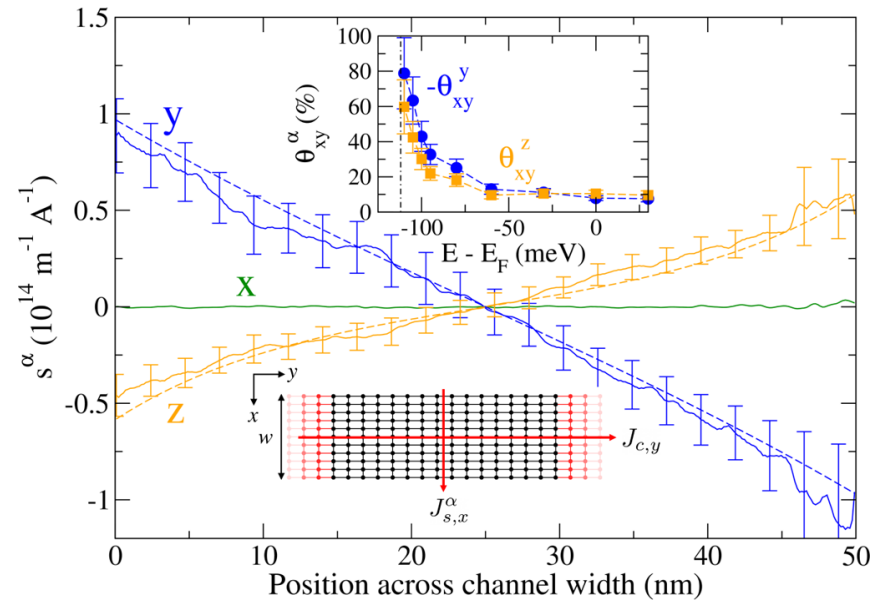

FIG. 3. Spin accumulation (solid lines) as a function of position across the channel width, of spins along $x, y$, and $z$. Error bars result from averaging over 200 disorder configurations $(w=$ $50 \mathrm{~nm}$ ). Dashed lines are fits to Eq. (2). Bottom inset: Scheme of the two-terminal device, where a current flowing along $y$ creates a spin accumulation in the $x$ direction. Top inset: Energy dependence of the spin Hall angles, with the conduction band minimum marked by a dot-dashed line.

Fig. 2 [49]. The results are displayed in the inset of Fig. 3. (We note that while the charge conductivity along $x$ and $y$ is slightly anisotropic, resulting in an equally anisotropic SHA, $\left|\theta_{x y}^{\alpha}\right|$ and $\left|\theta_{y x}^{\alpha}\right|$ are still very similar [49].) At $E_{F}$, the SHA for spins pointing along $y$ and $z$ is $\approx 10 \%$ (with opposite sign). Remarkably, both increase substantially when approaching the band edge, at which point $\left|\theta_{x y}^{y}\right|$ slightly overcomes $\left|\theta_{x y}^{z}\right|$ with values as large as $\left|\theta_{x y}^{y}\right| \approx 80 \%$. We also computed the SHC and the SHA with the Kubo formula and obtain the same result both qualitatively and quantitatively (supplementary Fig. 11). The increase of $\theta_{x y}$ is attributed to hot spots of SBC near the bottom of the electron pockets $[15,38]$ (Supplementary Figs. 4-6), which directly determine the SHC/SHA magnitude $[18,49,59,60]$. Importantly, our combined results

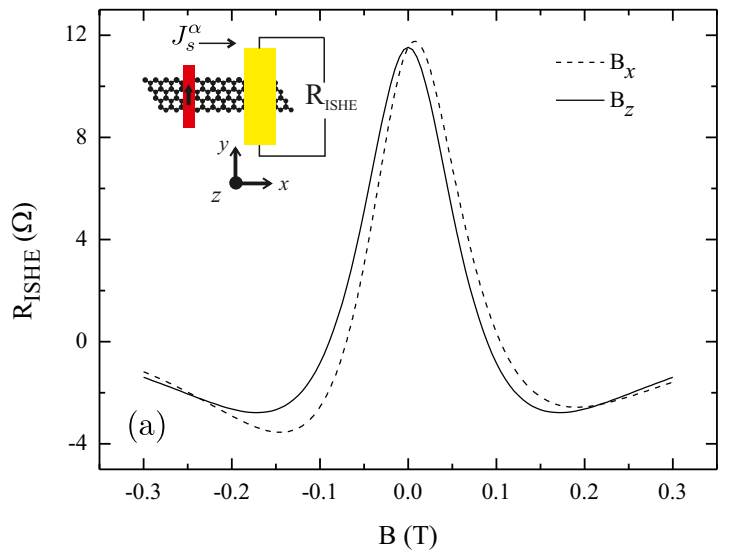

yield a CSI figure of merit $\lambda_{s} \theta_{x y} \approx 1-50 \mathrm{~nm}$, with the largest values attained at the band edge and for $y$-pointing spins. The upper limit exceeds that of traditional SOC materials ( $\mathrm{Pt}, \beta-\mathrm{W}$, $\beta$-Ta, or $\mathrm{Au}$ ) for which $\lambda_{s} \theta_{x y} \approx 0.1-0.2 \mathrm{~nm}[41,43,61]$, and is up to two to three times larger than that induced by proximity in graphene [62-64]. Such remarkable figure of merit stems from the combination of large SBC and the persistent spin texture near the $\mathrm{MoTe}_{2}$ band edges [49]. These results represent the expected behavior in the monolayer limit of recent experiments performed on few-layer $\mathrm{MoTe}_{2}[37,38]$ and $\mathrm{WTe}_{2}[39,40]$.

\section{EXPERIMENTAL DETECTION}

Such a peculiar spin response should become manifest in suitably designed nonlocal spin-precession experiments $[37,63,65,66]$. To probe this canted SHE, we propose the device concept pictured in the insets of Fig. 4, which relies on the reciprocal/inverse SHE (ISHE) [41]. It consists of a Hall bar comprising a graphene channel and a transversely aligned monolayer TMD crystal. A nonequilibrium spin accumulation is induced in the graphene channel through a FM electrode whose magnetization direction determines that of the spin density injected into graphene underneath. This generates a pure-spin current that diffuses toward-and is absorbed by - the remote TMD. It is assumed that the spin current is absorbed by the TMD at its edge and continues to follow the diffusion direction, given that the spin resistance in the TMD is two orders of magnitude lower than in graphene for $\lambda_{s}^{y}=30 \mathrm{~nm}$ or $\lambda_{s}^{z}=10 \mathrm{~nm}$ [49]. By ISHE, a transverse voltage $V_{\text {ISHE }}$ develops on the TMD, which can be measured along its length as illustrated in Fig. 4. In experiments, the diffusing spins can be controlled by spin precession in a noncollinear magnetic field $B$. To capture this situation, we generalized the Bloch diffusion equations to account for anisotropic spin diffusion and calculated $V_{\mathrm{ISHE}}(B)$ using the approach described in Ref. [63] (which accurately reproduces CSI in real devices). Figure 4 shows the precession response for two selected orientations of the TMD crystal in the limit of full absorption $\left(R_{\mathrm{ISHE}} \equiv V_{\mathrm{ISHE}} / I_{0}^{y}\right)$ [49]. We observe

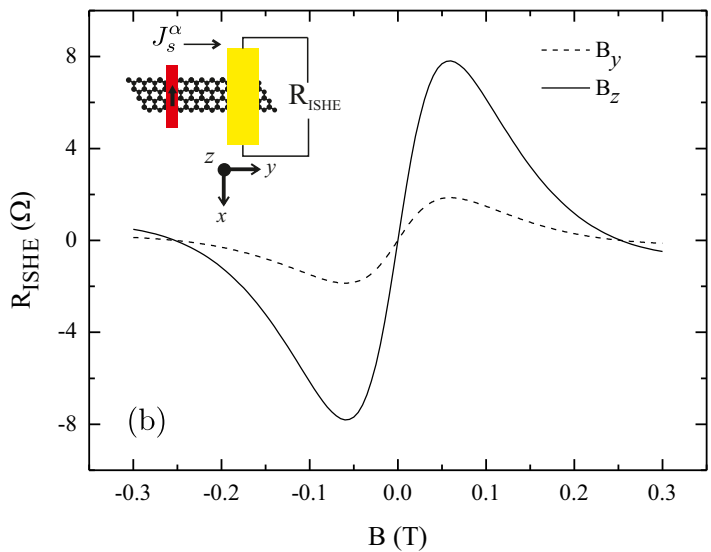

FIG. 4. Simulated response of the inverse SHE $\left(R_{\mathrm{ISHE}}\right)$ to spin precession for two orientations of the TMD crystal (coordinate axes in the insets). The device geometry is shown in the insets, with the TMD depicted in yellow and the FM injector in red (magnetization indicated by an arrow). The polarization of the spin current reaching the TMD $\left(\boldsymbol{J}_{s}^{\alpha}\right)$ is controlled externally with a magnetic field, $B$, oriented either along the graphene channel (dashed lines) or out-of-plane (solid lines). Typical experimental device dimensions were used in the simulation [49]. 
magnitudes of $R_{\text {ISHE }}$ nearly three orders of magnitude larger than the values reported for graphene/TMDs [62-64,67] and graphene/bulk-WSMs [37,39]. This is a direct consequence of the extremely large SHA predicted here for $\mathrm{MoTe}_{2}$ [68].

The essence of the experiment is that the precession response depends strongly on the crystal orientation. As evidenced in Fig. 2, the spin relaxation in the TMD is anisotropic, and the CSI depends crucially on both the majority spin orientation and crystalline orientation. In Fig. 4(a), the TMD's crystallographic $y$ axis is transverse to the spin propagation. A magnetic field parallel to $z$ causes spins to precess in the graphene plane but, according to Fig. 3, only the $y$ spin projection contributes to the ISHE signal with an efficiency of $\left|\theta_{x y}^{y}\right| ; R_{\mathrm{ISHE}}$ is symmetric with respect to the sign of $B$ because the magnetization at the FM injector is parallel to $y$, resulting in the maximum signal at $B=0$. When the field is parallel to $x$, the spins acquire a $z$ component (in addition to that in $y$ ), which is asymmetric with respect to $B$ and adds a contribution to the ISHE with an efficiency of $\left|\theta_{x y}^{z}\right|$; because $\left|\theta_{x y}^{y}\right| \gtrsim\left|\theta_{x y}^{z}\right|$, the signal remains roughly symmetric. In Fig. 4(b), the crystallographic $y$ axis is parallel to the spin propagation. As the $y$ and $z$ directions are now orthogonal to the FM magnetization, the line shapes are antisymmetric. The signal is zero at $B=0$ and, by sweeping $B$ from negative to positive along $z(x)$, the spin component along $y(z)$ changes sign. Therefore, two combined observations in this proposed experiment represent a "smoking gun" demonstration of the intrinsic canted SHE predicted in this work: (i) $R_{\mathrm{ISHE}}(B)$ should display a different line shape under different field orientations for a fixed TMD crystal; and (ii) rotation of the crystal converts the line shapes from predominantly symmetric to antisymmetric.

\section{GENERALIZATION TO OTHER SYSTEMS}

Our numerical calculations show that the $1 \mathrm{~T}_{\mathrm{d}}$ and $1 \mathrm{~T}^{\prime}$ phases of monolayer TMDs exhibit a canted spin Hall effect with large spin diffusion lengths and spin Hall angles. We precisely pinpoint this to the concurrence of a PST (which naturally enhances $\lambda_{s}$ ) and hot spots of SBC near the band edge. Additionally, the reduced symmetry has two fundamental consequences: It allows the extra nonzero components in the SHC tensor (hence a canted SHE) and it allows the PST (hence a large $\lambda_{s}$ ).

It is important to note that, though we focused here on the $\mathrm{MoTe}_{2}$ family in order to quantitatively demonstrate all the above features, the concurrence of PST and large SBC may now be expected in precisely identifiable space groups (SG). On the one hand, Tao and Tsymbal [69] have recently shown that robust PSTs (i.e., not relying on fine-tuned parameters [52]) likely arise in crystals belonging to some nonsymmorphic space groups; this symmetry-based approach can be extended to enumerate all compatible crystal families and the features of their allowed PSTs. On the other hand, crystal families compatible with SHC components other than $\sigma_{x y}^{z}$ have been also enumerated $[47,70]$, thereby identifying all the possible material platforms for canted, multicomponent SHE. Finally, the magnitude of intrinsic SHE depends directly on the strength of the SOC and on the existence of nonzero SBC (see our Supplemental Material'). Although both might be serendipitously large in some materials, topological ma- terials are preferred for a targeted pursuit. This is because SOC combined with the underlying band inversions of, e.g., topological insulators or topological semimetals, invariably leads to SBC hot spots $[22,71,72]$. Since space groups have also been recently classified according to their compatibility with different topological phases $[73,74]$, one may systematically select those topological classes whose symmetries are simultaneously compatible with PST and canted SHC.

To be more specific, we provide materials that satisfy the qualities mentioned above, thus making them potential candidates for showing large CSI figures of merit. We focused on materials from the space groups reported in Ref. [69] as they present PST. Although they only comprise orthorhombic crystals with no inversion symmetry, we note that extending the symmetry analysis of Ref. [69] to other crystal systems may provide greater number of potential SHE materials with PST. We searched these space groups in the Topological Material Database [74-77], and among the several topological insulators and semimetals, we searched which of those had already been experimentally characterized. At least three topological materials were found: the type-II Weyl semimetal $\mathrm{Ta}_{3} \mathrm{~S}_{2}$ (SG 39) [78] and $\mathrm{AuSn}_{4}$ (SG 41), which is a topological nodal-line semimetal [79]. According to the symmetry requirements for multicomponent SHE [47], the space groups from Ref. [69] in their bulk form cannot host a canted SHE. However, these restrictions are lifted in systems with glide mirrors and screw axis with vertical translation when going to the monolayer limit, as in $1 \mathrm{~T}_{\mathrm{d}}-\mathrm{MoTe}_{2}$. This is indeed the case for $\mathrm{TaIrTe}_{4}$ (SG 31), a van der Waals material being a Weyl semimetal in three dimensions but a topological insulator in monolayer form [80-82]. Overall, we have identified $\mathrm{Ta}_{3} \mathrm{~S}_{2}, \mathrm{AuSn}_{4}$, and $\mathrm{TaIrTe}_{4}$ as potential compounds for large CSI, with monolayer $\mathrm{TaIrTe}_{4}$ also being compatible with multicomponent SHE.

\section{POTENTIAL APPLICATIONS}

Discovering materials with largest $\lambda_{s} \theta_{x y}$ has been a long-standing challenge. This is partly because traditional understanding of SHE and spin diffusion posits that, while large SOC boosts the generation of spin current via SHE (quantified by $\theta_{x y}$ ), it detrimentally reduces the spin diffusion length [41]. Our extensive and realistic quantum simulations directly demonstrate that materials hosting low-symmetryenabled PST (even if only approximate) break free from that adverse compromise while, at the same time, displaying a new canted SHE, which greatly increases the geometrical flexibility of possible SHE-based devices. These results come at a time of impressive achievements in using 2D materials to carry spin currents over long distances and controlling their flow by electrostatic gating [83-85]. This should allow prompt exploration beyond our proof-of-principle system, $\mathrm{MoTe}_{2}$, thereby accelerating the potential delivery of low-power spinelectronic devices and circuits [86]. For example, the spin polarization generated by the canted SHE can exert an outof-plane antidamping torque in magnets with perpendicular magnetic anisotropy $[19,87,88]$, which are essential for nextgeneration, high-density spintronic applications [2,89]. 


\section{CONCLUSIONS}

Our models and quantum transport calculations of the spintronic response of $1 \mathrm{~T}_{\mathrm{d}}$ and $1 \mathrm{~T}^{\prime} \mathrm{MoTe}_{2}$ monolayers reveal the origin of a novel, canted SHE with long spin diffusion lengths, which reflects the unconventional spin textures allowed by their reduced symmetry and strong SOC. The obtained CSI figure of merit $\lambda_{s} \theta_{x y} \approx 1-50 \mathrm{~nm}$ is superior to that of traditional spintronic materials $(\mathrm{Pt}, \mathrm{Au}, \mathrm{W}$, and $\mathrm{Ta})[41,43]$ by up to two orders of magnitude. Given the similar electronic structures of $\mathrm{MoTe}_{2}$ and $\mathrm{WTe}_{2}$, including the persistent canted spin texture [90], comparable performance is expected in the latter. Our findings also call for a careful analysis of SHE measurements, since the interpretation of all-electrical detection in Hall bars $[38,91,92]$ usually ignores the possibility of multiple spin Hall components. We show how the presence of canted SHE can be experimentally identified by reciprocal SHE and how the different SHC contributions may be isolated in a spin precession setup.

Having precisely identified the underlying mechanism at play, these proof-of-principle results based on $\mathrm{MoTe}_{2}$ suggest equally promising performance in several other identifiable material families with concurrent PST and large spin Berry curvature associated with low crystal symmetry and nontrivial electronic topology, respectively. We finally mention that a much larger range of possible interesting materials should be available by engineering proximity effects and interfacial symmetries, as discussed for van der Waals heterostructures [93-95].

\section{ACKNOWLEDGMENTS}

M.V. acknowledges support from "La Caixa" Foundation and the Centre for Advanced 2D Materials at the National University of Singapore for its hospitality. X.W. acknowledges the Agence National pour la Recherche Flagera GRANSPORT funding. ICN2 authors were supported by the European Union Horizon 2020 research and innovation programme under Grant Agreements No. 881603 (Graphene Flagship) and No. 824140 (TOCHA, H2020FETPROACT-01-2018). ICN2 is funded by the CERCA Programme/Generalitat de Catalunya, and is supported by the Severo Ochoa program from Spanish Ministerio de Ciencia e Innovación (Grants No. SEV-2017-0706 and No. PID2019111773RB-I00/AEI/10.13039/501100011033). V.M.P. acknowledges the support of the National Research Foundation Singapore under its Medium-Sized Centre Programme.
[1] W. Han, Y. C. Otani, and S. Maekawa, Quantum materials for spin and charge conversion, npj Quantum Mater. 3, 27 (2018).

[2] A. Manchon, J. Železný, I. M. Miron, T. Jungwirth, J. Sinova, A. Thiaville, K. Garello, and P. Gambardella, Current-induced spin-orbit torques in ferromagnetic and antiferromagnetic systems, Rev. Mod. Phys. 91, 035004 (2019).

[3] K. Dolui, M. D. Petrovi, K. Zollner, P. Plech, J. Fabian, and B. K. Nikoli, Proximity spin-orbit torque on a two-dimensional magnet within van der Waals heterostructure: Currentdriven antiferromagnet-to-ferromagnet reversible nonequilibrium phase transition in bilayer $\mathrm{CrI}_{3}$, Nano Lett. 20, 2288 (2020).

[4] F. Bonell, M. Goto, G. Sauthier, J. F. Sierra, A. I. Figueroa, M. V. Costache, S. Miwa, Y. Suzuki, and S. O. Valenzuela, Control of spin-orbit torques by interface engineering in topological insulator heterostructures, Nano Lett. 20, 5893 (2020).

[5] Y. Fan and K. L. Wang, Spintronics based on topological insulators, SPIN 06, 1640001 (2016).

[6] A. Kononov, G. Abulizi, K. Qu, J. Yan, D. Mandrus, K. Watanabe, T. Taniguchi, and C. Schnenberger, Onedimensional edge transport in few-layer $\mathrm{WTe}_{2}$, Nano Lett. 20, 4228 (2020).

[7] A. A. Burkov and L. Balents, Weyl Semimetal in a Topological Insulator Multilayer, Phys. Rev. Lett. 107, 127205 (2011).

[8] X. Wan, A. M. Turner, A. Vishwanath, and S. Y. Savrasov, Topological semimetal and Fermi-arc surface states in the electronic structure of pyrochlore iridates, Phys. Rev. B 83, 205101 (2011).

[9] Y. Qi, P. G. Naumov, M. N. Ali, C. R. Rajamathi, W. Schnelle, O. Barkalov, M. Hanfland, S.-C. Wu, C. Shekhar, Y. Sun, V. Süß, M. Schmidt, U. Schwarz, E. Pippel, P. Werner, R. Hillebrand, T. Förster, E. Kampert, S. Parkin, R. J. Cava et al.,
Superconductivity in Weyl semimetal candidate $\mathrm{MoTe}_{2}$, Nat. Commun. 7, 11038 (2016).

[10] V. Fatemi, S. Wu, Y. Cao, L. Bretheau, Q. D. Gibson, K. Watanabe, T. Taniguchi, R. J. Cava, and P. Jarillo-Herrero, Electrically tunable low-density superconductivity in a monolayer topological insulator, Science 362, 926 (2018).

[11] E. Sajadi, T. Palomaki, Z. Fei, W. Zhao, P. Bement, C. Olsen, S Luescher, X. Xu, J. A. Folk, and D. H. Cobden, Gate-induced superconductivity in a monolayer topological insulator, Science 362, 922 (2018).

[12] K.-L. Chiu, D. Qian, J. Qiu, W. Liu, D. Tan, V. Mosallanejad, S. Liu, Z. Zhang, Y. Zhao, and D. Yu, Flux tunable superconducting quantum circuit based on Weyl semimetal $\mathrm{MoTe}_{2}$, Nano Lett. 20, 8469 (2020).

[13] Q. Ma, S.-Y. Xu, H. Shen, D. MacNeill, V. Fatemi, T.-R. Chang, A. M. Mier Valdivia, S. Wu, Z. Du, C.-H. Hsu, S. Fang, Q. D. Gibson, K. Watanabe, T. Taniguchi, R. J. Cava, E. Kaxiras, H.-Z. Lu, H. Lin, L. Fu, N. Gedik et al., Observation of the nonlinear Hall effect under time-reversal-symmetric conditions, Nature (London) 565, 337 (2019).

[14] K. Kang, T. Li, E. Sohn, J. Shan, and K. F. Mak, Nonlinear anomalous Hall effect in few-layer $\mathrm{WTe}_{2}$, Nat. Mater. 18, 324 (2019).

[15] S.-Y. Xu, Q. Ma, H. Shen, V. Fatemi, S. Wu, T.-R. Chang, G. Chang, A. M. M. Valdivia, C.-K. Chan, Q. D. Gibson, J. Zhou, Z. Liu, K. Watanabe, T. Taniguchi, H. Lin, R. J. Cava, L. Fu, N. Gedik, and P. Jarillo-Herrero, Electrically switchable Berry curvature dipole in the monolayer topological insulator $\mathrm{WTe}_{2}$, Nat. Phys. 14, 900 (2018).

[16] Y. Zhang, J. van den Brink, C. Felser, and B. Yan, Electrically tuneable nonlinear anomalous Hall effect in two-dimensional transition-metal dichalcogenides $\mathrm{WTe}_{2}$ and $\mathrm{MoTe}_{2}, 2 \mathrm{D}$ Mater. 5, 044001 (2018). 
[17] S. Singh, J. Kim, K. M. Rabe, and D. Vanderbilt, Engineering Weyl Phases and Nonlinear Hall Effects in $\mathrm{T}_{d}$-MoTe 2 , Phys. Rev. Lett. 125, 046402 (2020).

[18] J. Zhou, J. Qiao, A. Bournel, and W. Zhao, Intrinsic spin Hall conductivity of the semimetals, Phys. Rev. B 99, 060408(R) (2019).

[19] D. MacNeill, G. M. Stiehl, M. H. D. Guimaraes, R. A. Buhrman, J. Park, and D. C. Ralph, Control of spin-orbit torques through crystal symmetry in $\mathrm{WTe}_{2}$ /ferromagnet bilayers, Nat. Phys. 13, 300 (2017).

[20] P. Li, W. Wu, Y. Wen, C. Zhang, J. Zhang, S. Zhang, Z. Yu, S. A. Yang, A. Manchon, and X.-x. Zhang, Spin-momentum locking and spin-orbit torques in magnetic nano-heterojunctions composed of Weyl semimetal $\mathrm{WTe}_{2}$, Nat. Commun. 9, 3990 (2018).

[21] C. L. Kane and E. J. Mele, Quantum Spin Hall Effect in Graphene, Phys. Rev. Lett. 95, 226801 (2005).

[22] C. L. Kane and E. J. Mele, $Z_{2}$ Topological Order and the Quantum Spin Hall Effect, Phys. Rev. Lett. 95, 146802 (2005).

[23] X. Qian, J. Liu, L. Fu, and J. Li, Quantum spin Hall effect in two-dimensional transition metal dichalcogenides, Science $\mathbf{3 4 6}$, 1344 (2014).

[24] A. A. Soluyanov, D. Gresch, Z. Wang, Q. S. Wu, M. Troyer, X. Dai, and B. A. Bernevig, Type-II Weyl semimetals, Nature (London) 527, 495 (2015).

[25] Y. Sun, S.-C. Wu, M. N. Ali, C. Felser, and B. Yan, Prediction of Weyl semimetal in orthorhombic $\mathrm{MoTe}_{2}$, Phys. Rev. B 92, 161107(R) (2015).

[26] S. Tang, C. Zhang, D. Wong, Z. Pedramrazi, H.-Z. Tsai, C. Jia, B. Moritz, M. Claassen, H. Ryu, S. Kahn, J. Jiang, H. Yan, M. Hashimoto, D. Lu, R. G. Moore, C.-C. Hwang, C. Hwang, Z. Hussain, Y. Chen, M. M. Ugeda et al., Quantum spin Hall state in monolayer 1T'-WTe 2 , Nat. Phys. 13, 683 (2017).

[27] Z. Fei, T. Palomaki, S. Wu, W. Zhao, X. Cai, B. Sun, P. Nguyen, J. Finney, X. Xu, and D. H. Cobden, Edge conduction in monolayer $\mathrm{WTe}_{2}$, Nat. Phys. 13, 677 (2017).

[28] Z.-Y. Jia, Y.-H. Song, X.-B. Li, K. Ran, P. Lu, H.-J. Zheng, X.-Y. Zhu, Z.-Q. Shi, J. Sun, J. Wen, D. Xing, and S.-C. Li, Direct visualization of a two-dimensional topological insulator in the single-layer $1 T^{\prime}-\mathrm{WTe}_{2}$, Phys. Rev. B 96, 041108(R) (2017).

[29] P. Chen, W. Wu Pai, Y.-H. Chan, W.-L. Sun, C.-Z. Xu, D.-S. Lin, M. Y. Chou, A.-V. Fedorov, and T.-C. Chiang, Large quantum-spin-Hall gap in single-layer $1 \mathrm{~T}^{\prime} \mathrm{WSe}_{2}$, Nat. Commun. 9, 2003 (2018).

[30] L. Peng, Y. Yuan, G. Li, X. Yang, J.-J. Xian, C.-J. Yi, Y.-G. Shi, and Y.-S. Fu, Observation of topological states residing at step edges of $\mathrm{WTe}_{2}$, Nat. Commun. 8, 659 (2017).

[31] S. Wu, V. Fatemi, Q. D. Gibson, K. Watanabe, T. Taniguchi, R. J. Cava, and P. Jarillo-Herrero, Observation of the quantum spin Hall effect up to 100 kelvin in a monolayer crystal, Science 359, 76 (2018).

[32] Y. Shi, J. Kahn, B. Niu, Z. Fei, B. Sun, X. Cai, B. A. Francisco, D. Wu, Z.-X. Shen, X. Xu, D. H. Cobden, and Y.-T. Cui, Imaging quantum spin Hall edges in monolayer $\mathrm{WTe}_{2}$, Sci. Adv. 5, eaat8799 (2019).

[33] S.-Y. Xu, I. Belopolski, N. Alidoust, M. Neupane, G. Bian, C. Zhang, R. Sankar, G. Chang, Z. Yuan, C.-C. Lee, S.-M. Huang, H. Zheng, J. Ma, D. S. Sanchez, B. K. Wang, A. Bansil, F. Chou,
P. P. Shibayev, H. Lin, S. Jia et al., Discovery of a Weyl fermion semimetal and topological Fermi arcs, Science 349, 613 (2015).

[34] J. Jiang, Z. K. Liu, Y. Sun, H. F. Yang, C. R. Rajamathi, Y. P. Qi, L. X. Yang, C. Chen, H. Peng, C.-C. Hwang, S. Z. Sun, S.-K. Mo, I. Vobornik, J. Fujii, S. S. P. Parkin, C. Felser, B. H. Yan, and Y. L. Chen, Signature of type-II Weyl semimetal phase in $\mathrm{MoTe}_{2}$, Nat. Commun. 8, 13973 (2017).

[35] P. Li, Y. Wen, X. He, Q. Zhang, C. Xia, Z.-M. Yu, S. A. Yang, Z. Zhu, H. N. Alshareef, and X.-X. Zhang, Evidence for topological type-II Weyl semimetal $\mathrm{WTe}_{2}$, Nat. Commun. 8, 2150 (2017).

[36] C. Zhao, M. Hu, J. Qin, B. Xia, C. Liu, S. Wang, D. Dan Guan, Y. Li, H. Zheng, J. Liu, and J. Jia, Strain Tunable Semimetal-Topological-Insulator Transition in Monolayer $1 \mathrm{~T}^{\prime}-\mathrm{WTe}_{2}$, Phys. Rev. Lett. 125, 046801 (2020).

[37] C. K. Safeer, N. Ontoso, J. Ingla-Aynés, F. Herling, V. T. Pham, A. Kurzmann, K. Ensslin, A. Chuvilin, I. Robredo, M. G. Vergniory, F. de Juan, L. E. Hueso, M. R. Calvo, and F. Casanova, Large multidirectional spin-to-charge conversion in low-symmetry semimetal $\mathrm{MoTe}_{2}$ at room temperature, Nano Lett. 19, 8758 (2019).

[38] P. Song, C.-H. Hsu, G. Vignale, M. Zhao, J. Liu, Y. Deng, W. Fu, Y. Liu, Y. Zhang, H. Lin, V. M. Pereira, and K. P. Loh, Coexistence of large conventional and planar spin Hall effect with long spin diffusion length in a low-symmetry semimetal at room temperature, Nat. Mater. 19, 292 (2020).

[39] B. Zhao, D. Khokhriakov, Y. Zhang, H. Fu, B. Karpiak, A. Md. Hoque, X. Xu, Y. Jiang, B. Yan, and S. P. Dash, Observation of charge to spin conversion in Weyl semimetal $\mathrm{WTe}_{2}$ at room temperature, Phys. Rev. Research 2, 013286 (2020).

[40] B. Zhao, B. Karpiak, D. Khokhriakov, A. Johansson, A. Md. Hoque, X. Xu, Y. Jiang, I. Mertig, and S. P. Dash, Unconventional charge-spin conversion in Weyl-semimetal $\mathrm{WTe}_{2}, \mathrm{Adv}$ Mater. 32, 2000818 (2020).

[41] J. Sinova, S. O. Valenzuela, J. Wunderlich, C. H. Back, and T. Jungwirth, Spin Hall effects, Rev. Mod. Phys. 87, 1213 (2015).

[42] A. Hoffmann, Spin Hall effects in metals, IEEE Trans. Magn. 49, 5172 (2013).

[43] M. Isasa, E. Villamor, L. E. Hueso, M. Gradhand, and F. Casanova, Temperature dependence of spin diffusion length and spin Hall angle in Au and Pt, Phys. Rev. B 91, 024402 (2015).

[44] P. Laczkowski, Y. Fu, H. Yang, J.-C. Rojas-Sánchez, P. Noel, V. T. Pham, G. Zahnd, C. Deranlot, S. Collin, C. Bouard, P. Warin, V. Maurel, M. Chshiev, A. Marty, J.-P. Attané, A. Fert, H. Jaffrès, L. Vila, and J.-M. George, Large enhancement of the spin Hall effect in Au by side-jump scattering on Ta impurities, Phys. Rev. B 96, 140405(R) (2017).

[45] E. Sagasta, Y. Omori, S. Vélez, R. Llopis, C. Tollan, A. Chuvilin, L. E. Hueso, M. Gradhand, Y.-C. Otani, and F. Casanova, Unveiling the mechanisms of the spin Hall effect in Ta, Phys. Rev. B 98, 060410(R) (2018).

[46] S. Sayed, S. Hong, X. Huang, L. Caretta, A. S. Everhardt, R. Ramesh, S. Salahuddin, and S. Datta, Unified Framework for Charge-Spin Interconversion in Spin-Orbit Materials, Phys. Rev. Appl. 15, 054004 (2021).

[47] M. Seemann, D. Ködderitzsch, S. Wimmer, and H. Ebert, Symmetry-imposed shape of linear response tensors, Phys. Rev. B 92, 155138 (2015). 
[48] Z. Fan, J. H. Garcia, A. W. Cummings, J. E. Barrios-Vargas, M. Panhans, A. Harju, F. Ortmann, and S. Roche, Linear scaling quantum transport methodologies, Phys. Rep. 903, 1 (2021).

[49] See Supplemental Material at http://link.aps.org/supplemental/ 10.1103/PhysRevResearch.3.043230 for a detailed explanation of the tight-binding model, the DFT calculations, the spin Berry curvature of the bands, the setup for the Landauer-Büttiker and Kubo-Bastin simulations, the derivation of Eq. (2), the relation between the (persistent) spin texture and spin relaxation, the spin transport for in-gap states, the results for the $1 \mathrm{~T}^{\prime}$ phase, and the modeling of the experimental detection of the canted SHE.

[50] J. Schliemann, J. C. Egues, and D. Loss, Nonballistic SpinField-Effect Transistor, Phys. Rev. Lett. 90, 146801 (2003).

[51] B. A. Bernevig, J. Orenstein, and S.-C. Zhang, Exact SU(2) Symmetry and Persistent Spin Helix in a Spin-Orbit Coupled system, Phys. Rev. Lett. 97, 236601 (2006).

[52] J. Schliemann, Colloquium: Persistent spin textures in semiconductor nanostructures, Rev. Mod. Phys. 89, 011001 (2017).

[53] L.-k. Shi and J. C. W. Song, Symmetry, spin texture, and tunable quantum geometry in a $\mathrm{WTe}_{2}$ monolayer, Phys. Rev. B 99, 035403 (2019).

[54] C. W. Groth, M. Wimmer, A. R. Akhmerov, and X. Waintal, KWANT: A software package for quantum transport, New J. Phys. 16, 063065 (2014).

[55] M. Johnson and R. H. Silsbee, Interfacial Charge-Spin Coupling: Injection and Detection of Spin Magnetization in Metals, Phys. Rev. Lett. 55, 1790 (1985).

[56] F. J. Jedema, H. B. Heersche, A. T. Filip, J. J. A. Baselmans, and B. J. Van Wees, Electrical detection of spin precession in a metallic mesoscopic spin valve, Nature (London) 416, 713 (2002).

[57] J. Fabian, A. Matos-Abiaguea, P. Ertler, C. snd Stano, and I. Žutić, Semiconductor spintronics, Acta Phys. Slovaca 57, 565 (2007).

[58] M. Vila, J. H. Garcia, A. W. Cummings, S. R. Power, C. W. Groth, X. Waintal, and S. Roche, Nonlocal Spin Dynamics in the Crossover from Diffusive to Ballistic Transport, Phys. Rev. Lett. 124, 196602 (2020).

[59] J. Sinova, D. Culcer, Q. Niu, N. A. Sinitsyn, T. Jungwirth, and A. H. MacDonald, Universal Intrinsic Spin Hall Effect, Phys. Rev. Lett. 92, 126603 (2004).

[60] T. Tanaka, H. Kontani, M. Naito, T. Naito, D. S. Hirashima, K. Yamada, and J. Inoue, Intrinsic spin Hall effect and orbital Hall effect in in 4d and 5d transition metals, Phys. Rev. B 77, 165117 (2008).

[61] K. Roy, Estimating the spin diffusion length and the spin Hall angle from spin pumping induced inverse spin Hall voltages, Phys. Rev. B 96, 174432 (2017).

[62] C. K. Safeer, J. Ingla-Aynés, F. Herling, J. H. Garcia, M. Vila, N. Ontoso, M. R. Calvo, S. Roche, L. E. Hueso, and F. Casanova, Room-temperature spin Hall effect in graphene/ $\mathrm{MoS}_{2}$ van der Waals heterostructures, Nano Lett. 19, 1074 (2019).

[63] L. A. Benítez, W. Savero Torres, J. F. Sierra, M. Timmermans, J. H. Garcia, S. Roche, M. V. Costache, and S. O. Valenzuela, Tunable room-temperature spin galvanic and spin Hall effects in van der Waals heterostructures, Nat. Mater. 19, 170 (2020).
[64] F. Herling, C. K. Safeer, J. Ingla-Ayns, N. Ontoso, L. E. Hueso, and F. Casanova, Gate tunability of highly efficient spin-tocharge conversion by spin Hall effect in graphene proximitized with $\mathrm{WSe}_{2}$, APL Mater. 8, 071103 (2020).

[65] W. Savero Torres, J. F. Sierra, L. A. Benítez, F. Bonell, M. V. Costache, and S. O. Valenzuela, Spin precession and spin Hall effect in monolayer graphene/Pt nanostructures, 2D Mater. 4, 041008 (2017).

[66] S. A. Cavill, C. Huang, M. Offidani, Y.-H. Lin, M. A. Cazalilla, and A. Ferreira, Proposal for Unambiguous Electrical Detection of Spin-Charge Conversion in Lateral Spin Valves, Phys. Rev. Lett. 124, 236803 (2020).

[67] T. S. Ghiasi, A. A. Kaverzin, P. J. Blah, and B. J. van Wees, Charge-to-spin conversion by the Rashba-Edelstein effect in two-dimensional van der Waals heterostructures up to room temperature, Nano Lett. 19, 5959 (2019).

[68] In experiments, $R_{\mathrm{ISHE}}$ also depends on the TMD's electrical resistance [49] and the Fermi level, as seen in Fig. 3. Interfacial barriers between graphene and the TMD can suppress the spin sink effect and cause a more distributed spin absorption across the TMD width, in which case the observed signal might be partially reduced.

[69] L. L. Tao and E. Y. Tsymbal, Persistent spin texture enforced by symmetry, Nat. Commun. 9, 2763 (2018).

[70] Y. Zhang, Q. Xu, K. Koepernik, R. Rezaev, O. Janson, J. Železný, T. Jungwirth, C. Felser, J. van den Brink, and Y. Sun, Different types of spin currents in the comprehensive materials database of nonmagnetic spin Hall effect, npj Comput. Mater. 7, 167 (2021).

[71] Y. Sun, Y. Zhang, C. Felser, and B. Yan, Strong intrinsic spin Hall effect in the TaAs family of Weyl semimetals, Phys. Rev. Lett. 117, 146403 (2016).

[72] N. H. D. Khang, Y. Ueda, and P. Nam Hai, A conductive topological insulator with large spin Hall effect for ultralow power spin-orbit torque switching, Nat. Mater. 17, 808 (2018).

[73] T. Zhang, Y. Jiang, Z. Song, H. Huang, Y. He, Z. Fang, H. Weng, and C. Fang, Catalogue of topological electronic materials, Nature (London) 566, 475 (2019).

[74] M. G. Vergniory, L. Elcoro, C. Felser, N. Regnault, B. A. Bernevig, and Z. Wang, A complete catalogue of high-quality topological materials, Nature (London) 566, 480 (2019).

[75] B. Bradlyn, L. Elcoro, J. Cano, M. G. Vergniory, Z. Wang, C. Felser, M. I. Aroyo, and B. A. Bernevig, Topological quantum chemistry, Nature (London) 547, 298 (2017).

[76] M. G. Vergniory, B. J. Wieder, L. Elcoro, S. S. P. Parkin, C. Felser, B. A. Bernevig, and N. Regnault, All topological bands of all stoichiometric materials, arXiv:2105.09954.

[77] See https://topologicalquantumchemistry.org and https://www. cryst.ehu.es.

[78] G. Chang, S.-Y. Xu, D. S. Sanchez, S.-M. Huang, C.-C. Lee, T.R. Chang, G. Bian, H. Zheng, I. Belopolski, N. Alidoust, H.-T. Jeng, A. Bansil, H. Lin, and M. Z. Hasan, A strongly robust type II Weyl fermion semimetal state in $\mathrm{Ta}_{3} \mathrm{~S}_{2}$, Sci. Adv. 2, e1600295 (2016).

[79] D. Shen, C. N. Kuo, T. W. Yang, I. N. Chen, C. S. Lue, and L. M. Wang, Two-dimensional superconductivity and magnetotransport from topological surface states in $\mathrm{AuSn}_{4}$ semimetal, Commun. Mater. 1, 56 (2020).

[80] E. Haubold, K. Koepernik, D. Efremov, S. Khim, A. Fedorov, Y. Kushnirenko, J. van den Brink, S. Wurmehl, B. Büchner, 
T. K. Kim, M. Hoesch, K. Sumida, K. Taguchi, T. Yoshikawa, A. Kimura, T. Okuda, and S. V. Borisenko, Experimental realization of type-II Weyl state in noncentrosymmetric TaIrTe ${ }_{4}$, Phys. Rev. B 95, 241108(R) (2017).

[81] I. Belopolski, P. Yu, D. S. Sanchez, Y. Ishida, T.-R. Chang, S. S. Zhang, S.-Y. Xu, H. Zheng, G. Chang, G. Bian, H.-T. Jeng, T. Kondo, H. Lin, Z. Liu, S. Shin, and M. Zahid Hasan, Signatures of a time-reversal symmetric Weyl semimetal with only four Weyl points, Nat. Commun. 8, 942 (2017).

[82] J. Liu, H. Wang, C. Fang, L. Fu, and X. Qian, van der Waals stacking-induced topological phase transition in layered ternary transition metal chalcogenides, Nano Lett. 17, 467 (2017).

[83] W. Yan, O. Txoperena, R. Llopis, H. Dery, L. E. Hueso, and F. Casanova, A two-dimensional spin field-effect switch, Nat. Commun. 7, 13372 (2016).

[84] A. Dankert and S. P. Dash, Electrical gate control of spin current in van der Waals heterostructures at room temperature, Nat. Commun. 8, 16093 (2017).

[85] X. Lin, L. Su, Z. Si, Y. Zhang, A. Bournel, Y. Zhang, J.-O. Klein, A. Fert, and W. Zhao, Gate-Driven Pure Spin Current in Graphene, Phys. Rev. Appl. 8, 034006 (2017).

[86] X. Lin, W. Yang, K. L. Wang, and W. Zhao, Two-dimensional spintronics for low-power electronics, Nat. Electron. 2, 274 (2019).

[87] D. MacNeill, G. M. Stiehl, M. H. D. Guimarães, N. D. Reynolds, R. A. Buhrman, and D. C. Ralph, Thickness dependence of spin-orbit torques generated by $\mathrm{WTe}_{2}$, Phys. Rev. B 96, 054450 (2017).

[88] G. M. Stiehl, R. Li, V. Gupta, I. El Baggari, S. Jiang, H. Xie, L. F. Kourkoutis, K. F. Mak, J. Shan, R. A. Buhrman, and
D. C. Ralph, Layer-dependent spin-orbit torques generated by the centrosymmetric transition metal dichalcogenide $\beta-\mathrm{MoTe}_{2}$, Phys. Rev. B 100, 184402 (2019).

[89] Y. Liu and Q. Shao, Two-dimensional materials for energyefficient spin-orbit torque devices, ACS Nano 14, 9389 (2020).

[90] J. H. Garcia, M. Vila, C.-H. Hsu, X. Waintal, V. M. Pereira, and S. Roche, Canted Persistent Spin Texture and Quantum Spin Hall Effect in $\mathrm{WTe}_{2}$, Phys. Rev. Lett. 125, 256603 (2020).

[91] D. A. Abanin, A. V. Shytov, L. S. Levitov, and B. I. Halperin, Nonlocal charge transport mediated by spin diffusion in the spin Hall effect regime, Phys. Rev. B 79, 035304 (2009).

[92] E. M. Hankiewicz, L. W. Molenkamp, T. Jungwirth, and J. Sinova, Manifestation of the spin Hall effect through chargetransport in the mesoscopic regime, Phys. Rev. B 70, 241301(R) (2004).

[93] J. F. Sierra, J. Fabian, R. K. Kawakami, S. Roche, and S. O. Valenzuela, Van der Waals heterostructures for spintronics and opto-spintronics, Nat. Nanotechnol. 16, 856 (2021).

[94] H. Kurebayashi, J. H. Garcia, S. Khan, J. Sinova, and S. Roche, Magnetism, symmetry, and spin transport in van der Waals layered systems, arXiv:2107.03763 [Nat. Rev. Phys. (to be published)].

[95] F. Giustino, J. H. Lee, F. Trier, M. Bibes, S. M. Winter, R. Valentí, Y.-W. Son, L. Taillefer, C. Heil, A. I. Figueroa, B. Plaçais, Q. S. Wu, O. V. Yazyev, E. P. A. M. Bakkers, J. Nygård, P. Forn-Díaz, S. De Franceschi, J. W. McIver, L. E. F. Foa Torres, T. Low, A. Kumar, R. Galceran, S. O. Valenzuela, M. V. Costache, A. Manchon, E.-A. Kim, G. R. Schleder, A. Fazzio, and S. Roche, The 2021 quantum materials roadmap, J. Phys. Mater. 3, 042006 (2020). 